\title{
On a conjecture of G. Malle and G. Navarro on nilpotent blocks
}

\author{
Jean-Baptiste Gramain \\ Institute of Mathematics \\ University of Aberdeen \\ King's College, Fraser Noble Building \\ Aberdeen, AB24 3UE, UK \\ jbgramain@abdn.ac.uk
}

Submitted: June 1, 2011; Accepted: Oct 27, 2011; Published: Nov 7, 2011

Mathematics Subject Classification: 20C30 (primary), 20C15, 20C20 (secondary)

\begin{abstract}
In a recent article, G. Malle and G. Navarro conjectured that the $p$-blocks of a finite group all of whose height 0 characters have the same degree are exactly the nilpotent blocks defined by M. Broué and L. Puig. In this paper, we check that this conjecture holds for spin-blocks of the covering group $2 . \mathfrak{A}_{n}$ of the alternating group $\mathfrak{A}_{n}$, thereby solving a case excluded from the study of quasi-simple groups by Malle and Navarro.
\end{abstract}

\section{Introduction}

In a recent paper ([3]), G. Malle and G. Navarro have formulated a conjecture about nilpotent blocks of finite groups. The notion of nilpotent block was first introduced by M. Broué and L. Puig in [1], and should be the most natural to study from a local point of view. However, the definition given by Broué and Puig uses the Alperin-Broué subpairs, making the detection of nilpotent blocks a difficult problem. One strong property of nilpotent blocks is that, if a $p$-block $B$ of a finite group $G$ is nilpotent, then all the height zero characters $\chi \in \operatorname{Irr}_{0}(B)$ have the same degree. In [3], Malle and Navarro conjecture that the converse also holds, therefore giving a global characterization of nilpotent blocks which is visible in the character table of $G$.

In their paper, Malle and Navarro prove that their conjecture is true whenever $B$ is the principal block of $G([3$, Theorem 3.1]), or if the defect group $D$ of $B$ is normal in $G$ ([3,

Keywords: Representation Theory, Symmetric Group, Covering Groups, Bar-Partitions

THE ELECTRONIC JOURNAL OF COMBINATORICS 18 (2011), \#P217 
Theorem 5.2]). They also prove that it holds whenever $D$ is abelian, provided Brauer's Height Zero Conjecture holds ([3, Theorem 4.1]), and make considerable progress in the case of $p$-solvable groups. Finally, they give a proof of their conjecture for all finite quasisimple groups ([3, Theorem 6.1]), with the possible exception of quasi-isolated blocks of exceptional groups of Lie type in bad characteristic, and faithful blocks of the 2-fold covering group $2 . \mathfrak{A}_{n}$ of the alternating group $\mathfrak{A}_{n}(n \geq 14)$.

The object of this paper is to prove that the covering group $2 . \mathfrak{A}_{n}$ does not in fact yield any counter-example to the conjecture of Malle and Navarro, i.e. that any block of $2 . \mathfrak{A}_{n}$ all of whose height zero characters have the same degree is nilpotent (Corollary 4.2). In Section 2, we introduce the classical results about characters and blocks we need to study the case of $2 \cdot \mathfrak{A}_{n}$. In Section 3, we construct, for blocks of $2 . \mathfrak{S}_{n}$ with non-abelian defect group, height zero characters with distinct degrees. Finally, Section 4 is devoted to restricting these characters to $2 . \mathfrak{A}_{n}$ and checking that they do provide the desired result in this case.

Note that, even though our method is analogous to that used by Malle and Navarro in the case of $\mathfrak{A}_{n}$, the fact that we use bar-partitions and bars instead of partitions and hooks induces several complications. Also, there is no clear bar-analogue of the relative hook-formula for character degrees they use in the symmetric group.

\section{Characters and blocks of covering groups}

In this section, we present an overview of the representation theory of the covering groups of $\mathfrak{S}_{n}$ and $\mathfrak{A}_{n}$. These groups were first introduced and studied by I. Schur in [6]. Unless stated otherwise, all the results in this section (and references for proofs) can found for example in [5].

The symmetric group $\mathfrak{S}_{n}$ has, for $n \geq 4$, two non-isomorphic 2-fold covering groups (only one if $n=6$ ), which have the same character table. We therefore denote, slightly abusively, by $2 . \mathfrak{S}_{n}$ one of these covering groups. Then $2 . \mathfrak{S}_{n}$ has center $\langle z\rangle$ of order 2 , and $2 . \mathfrak{S}_{n} /\langle z\rangle \cong \mathfrak{S}_{n}$. The group 2. $\mathfrak{S}_{n}$ has a (unique, normal) subgroup of index 2, which is the unique 2 -fold covering group $2 . \mathfrak{A}_{n}$ of the alternating group $\mathfrak{A}_{n}$.

The irreducible complex characters $\chi$ of $2 . \mathfrak{S}_{n}$ and $2 . \mathfrak{A}_{n}$ fall into two categories. If $z \in \operatorname{ker}(\chi)$, then $\chi$ is just lifted from an irreducible character of $\mathfrak{S}_{n}$ or $\mathfrak{A}_{n}$. Otherwise, $\chi$ is a faithful character, also called spin-character, and corresponds to a projective representation of $\mathfrak{S}_{n}$ or $\mathfrak{A}_{n}$.

The spin-characters of $2 . \mathfrak{S}_{n}$ and $2 . \mathfrak{A}_{n}$ are canonically labelled by the bar-partitions of $n$, i.e. partitions of $n$ in distinct parts. If $\lambda=\left(a_{1}>\cdots>a_{m}>0\right)$ is a bar-partition of $n$, then we let $m(\lambda)=m$ and $\sigma(\lambda)=(-1)^{n-m(\lambda)}$. If $\sigma(\lambda)=1$, then $\lambda$ labels a unique spincharacter of $2 . \mathfrak{S}_{n}$. The restriction to $2 . \mathfrak{A}_{n}$ of this character is the sum of two associate spin-characters, both labelled by $\lambda$. If, on the other hand, $\sigma(\lambda)=-1$, then $\lambda$ labels two associate spin-characters of $2 . \mathfrak{A}_{n}$; both have the same restriction to $2 . \mathfrak{A}_{n}$, which is the unique spin-character of $2 . \mathfrak{A}_{n}$ labelled by $\lambda$. 
The notion that replaces that of hooks in partitions is given by bars in bar-partitions. If $\lambda=\left(a_{1}>\cdots>a_{m}>0\right)$ is a bar-partition of $n$, then the set of bar-lengths in $\lambda$ is

$$
\overline{\mathcal{H}}(\lambda)=\bigcup_{1 \leq i \leq m}\left\{1, \ldots, a_{i}\right\} \cup\left\{a_{i}+a_{j} \mid j>i\right\} \backslash\left\{a_{i}-a_{j} \mid j>i\right\}
$$

For any integer $\ell$, we call $\bar{\ell}$-weight of $\lambda$ the number of bars in $\lambda$ whose length is divisible by $\ell$. Such a bar is called an $(\ell)$-bar.

Writing $\bar{H}(\lambda)$ for the product of all the bar-lengths in $\lambda$, we have, in analogy with the Hook-Length Formula for the degree of characters of $\mathfrak{S}_{n}$, that any spin-character of $2 . \mathfrak{S}_{n}$ labelled by $\lambda$ has degree $2^{\lfloor(n-m(\lambda)) / 2\rfloor} \frac{n !}{\bar{H}(\lambda)}$.

If we now take a prime $p$, then the distribution of irreducible characters of $2 . \mathfrak{S}_{n}$ and $2 . \mathfrak{A}_{n}$ into $p$-blocks depends on the parity of $p$.

If $p$ is odd, then every $p$-block $B$ of $2 . \mathfrak{S}_{n}$ or $2 . \mathfrak{A}_{n}$ contains either no spin-character, or only spin-characters. A block with no spin-character is really just a block of $\mathfrak{S}_{n}$ or $\mathfrak{A}_{n}$, and its defect groups are the same as in these groups. In particular, the conjecture of Malle and Navarro holds for these blocks of $2 . \mathfrak{A}_{n}$ because it holds in $\mathfrak{A}_{n}$ (by [3, Corollary 9.3]). If $B$ contains only spin-characters, then $B$ is refered to as a spin-block, or faithful block. Such a block has either defect 0 , hence contains a unique spin-character (labelled by a bar-partition with $\bar{p}$-weight 0 ), or it consists exactly of all the spin-characters labelled by bar-partitions with a given $\bar{p}$-core (the bar-partition obtained by removing from a barpartition all the bars of length divisible by $p$ ). This is known as the Morris Conjecture, which is the analogue of the Nakayama Conjecture for the symmetric group. Each spinblock $B$ of $2 . \mathfrak{S}_{n}$ of $\bar{p}$-weight $w \geq 1$ covers a unique block $B^{*}$ of $2 . \mathfrak{A}_{n}$ which is labelled by the same $\bar{p}$-core (and each spin-block of $2 . \mathfrak{A}_{n}$ is covered by a unique spin-block of $2 . \mathfrak{S}_{n}$ ). The defect groups of $B$ and $B^{*}$ are entirely determined by $w$, and are the same as the defect groups of any block of $p$-weight $w$ in $\mathfrak{S}_{n}$. We also see from the degree formula that a spin-character of $B$ has height 0 if and only if it's labelled by a bar-partition of ( $\bar{p}$-weight $w$ and) maximal $\bar{p}^{2}$-weight, maximal $\bar{p}^{3}$-weight, ...

If, on the other hand, $p=2$, then each $p$-block of positive defect of $2 . \mathfrak{S}_{n}$ or $2 . \mathfrak{A}_{n}$ contains both spin-characters and non-spin-characters. To check that Malle and Navarro's conjecture holds in this case, it is thus sufficient to check that, if such a block has nonabelian defect, then it contains two non-spin-characters of height 0 with distinct degrees. But, by [3, Corollary 9.3], any non-spin-block $B$ of $2 . \mathfrak{A}_{n}$ all of whose height zero nonspin-characters have the same degree would correspond to a block $b$ of $\mathfrak{A}_{n}$ with the same property, and thus of weight 0 in $\mathfrak{A}_{n}$, so that $B$ has (abelian) defect group of order 2 in $2 . \mathfrak{A}_{n}$.

From now on, we therefore always suppose that $p$ is an odd prime, and only consider spin-blocks. 


\section{Bar-partitions with different products of bar- lengths}

Take any odd prime $p$, and let $\gamma$ be a $\bar{p}$-core. Until the last result of this section, we assume furthermore that $\gamma \neq \emptyset$.

We write $\gamma=\left(a_{1}>\cdots>a_{m}>0\right)$ and $X_{\gamma}=\left\{a_{1}, \ldots, a_{m}\right\}$. The bars in $\gamma$ correspond to

- pairs $(x, y)$, with $0 \leq x<y, x \notin X_{\gamma}$ and $y \in X_{\gamma}$ (these have length $y-x$, and type 2 if $x=0$ and type 1 if $x \neq 0$ ), and

- $\operatorname{pairs}(i, j)$, with $1 \leq i<j \leq m$ (these have length $a_{i}+a_{j}$ and type 3 ).

We start by giving three easy consequences of the fact that $\gamma$ is a $\bar{p}$-core. Firstly, for all $1 \leq i \leq m$, we have $a_{i} \not \equiv 0(\bmod p)$ (otherwise, $\gamma$ would have a $(p)$-bar of type 2$)$. We can thus arrange the $a_{i}$ 's according to their value $\bmod p$, and let

$$
X_{0}=\left\{a_{i} \mid a_{i} \equiv 0(\bmod p)\right\}=\emptyset \text { and } X_{j}=\left\{a_{i} \mid a_{i} \equiv j(\bmod p)\right\} \text { for } 1 \leq j \leq p-1 .
$$

If $X_{j} \neq \emptyset$, then $X_{p-j}=\emptyset$ (otherwise, we would have $a_{t}=p k_{t}+j \in X_{j}$ and $a_{s}=$ $p k_{s}+(p-j) \in X_{p-j}$, and thus the $(p)$-bar $a_{t}+a_{s}=p\left(k_{t}+k_{s}+1\right)$ of type 2$)$. Finally, if $X_{j} \neq \emptyset$, then $X_{j}=\left\{j+k p, 0 \leq k \leq b_{j}\right\}$. Indeed:

- $j \in X_{j}$ since, otherwise, $\left(j, a_{i}\right)$ (for any $a_{i} \in X_{j}$ ) would give a $(p)$-bar of type 1 (Note that, obviously, if $j \in X_{\gamma}$, then $j \in X_{j}$ );

- if $j+s p \in X_{j}$ for some $s>0$, then $j+t p \in X_{j}$ for all $0 \leq t<s$ (since, otherwise, $(j+t p, j+s p)$ would give a $(p)$-bar of type 1$)$.

If $X_{j}=\emptyset$, then we set $b_{j}=-1$. Note that our definition of $b_{j}$ differs from that of Malle and Navarro (see [3, Theorem 9.1]), which is the number of beads on the $j$-th runner of the $p$-abacus of $\gamma^{*}$, the partition (which is a $p$-core) corresponding to the $\beta$-set $X_{\gamma}$. Our $b_{j}$ is always exactly 1 less.

For any $0 \leq j \leq p-1$, we also write $c_{j}=j+b_{j} p$. Here again, our definition differs from that of Malle and Navarro.

Now take any integer $w \geq 1$. We define some bar-partitions with $\bar{p}$-core $\gamma$ and $\bar{p}$-weight $w$.

- Define $\lambda_{0}^{(w)}$ by letting $X_{\lambda_{0}^{(w)}}=X_{\gamma} \cup\{p w\}=\bigcup_{j=0}^{p-1} X_{j}^{0,(w)}$ (so that $X_{0}^{0,(w)}=\{p w\}$ and $X_{j}^{0,(w)}=X_{j}$ for $\left.1 \leq j \leq p-1\right)$. Note that $\lambda_{0}^{(w)}$ can be defined even if $\gamma=\emptyset$.

- If $X_{i} \neq \emptyset$ (for some $1 \leq i \leq p-1$ ), then define $\lambda_{i}^{(w)}$ by letting $X_{\lambda_{i}^{(w)}}=X_{\gamma} \cup\left\{i+b_{i} p+\right.$ $p w\} \backslash\left\{i+b_{i} p\right\}=\bigcup_{j=0}^{p-1} X_{j}^{i,(w)}$ (so that $X_{0}^{i,(w)}=\emptyset, X_{j}^{i,(w)}=X_{j}$ for $1 \leq j \neq i \leq p-1$, and $\left.X_{i}^{i,(w)}=X_{i} \cup\left\{i+b_{i} p+p w\right\} \backslash\left\{i+b_{i} p\right\}\right)$. 
Note that $\lambda_{0}^{(w)}$ and (if $\left.X_{i} \neq \emptyset\right) \lambda_{i}^{(w)}$ are bar-partitions of $|\gamma|+p w$, with $\bar{p}$-weight at least $w$ and $\bar{p}$-core $\gamma$, and thus $\bar{p}$-weight exactly $w$.

Note also that, by definition, all the $(p)$-bars in $\lambda_{0}^{(w)}$ are of type 1 or of type 2 (and there's exactly one of these), while, if $X_{i} \neq \emptyset$, then all the $(p)$-bars in $\lambda_{i}^{(w)}$ are of type 1 .

Finally, note that $m\left(\lambda_{0}^{(w)}\right)=m(\gamma)+1$, while, if $X_{i} \neq \emptyset$, then $m\left(\lambda_{i}^{(w)}\right)=m(\gamma)$.

First, we suppose that $X_{i} \neq \emptyset$, and we want to compare $\bar{H}\left(\lambda_{i}^{(w)}\right)$ and $\bar{H}\left(\lambda_{i}^{(w-1)}\right)$ (the products of all bar-lengths in $\lambda_{i}^{(w)}$ and $\lambda_{i}^{(w-1)}$ respectively). Note that, if $w=1$, then $\lambda_{i}^{(w-1)}=\gamma$. Write $\bar{H}\left(\lambda_{i}^{(w)}\right)=\bar{H}_{m}\left(\lambda_{i}^{(w)}\right) \bar{H}_{u}\left(\lambda_{i}^{(w)}\right)$ and $\bar{H}\left(\lambda_{i}^{(w-1)}\right)=\bar{H}_{m}\left(\lambda_{i}^{(w-1)}\right) \bar{H}_{u}\left(\lambda_{i}^{(w-1)}\right)$, where $\bar{H}_{m}$ (respectively $\bar{H}_{u}$ ) stands for the product of all mixed (respectively unmixed) bar-lengths, i.e. of type 2 or 3 (respectively of type 1 ).

Proposition 3.1. With the above notation, we have

$$
\frac{\bar{H}_{u}\left(\lambda_{i}^{(w)}\right)}{\bar{H}_{u}\left(\lambda_{i}^{(w-1)}\right)}=p w \prod_{0 \leq j \neq i \leq p-1}\left|p(w-1)+c_{i}-c_{j}\right|
$$

and

$$
\frac{\bar{H}_{m}\left(\lambda_{i}^{(w)}\right)}{\bar{H}_{m}\left(\lambda_{i}^{(w-1)}\right)}=\prod_{j \neq i, X_{j} \neq \emptyset} \frac{c_{i}+c_{j}+p w}{c_{i}+p(w-1)+j} .
$$

Proof. To prove the first part, one can simply compare explicitly all the unmixed bars in $\lambda_{i}^{(w)}$ and $\lambda_{i}^{(w-1)}$. Another, more elegant way, is to apply [3, Theorem 9.1] (or the special case that immediately follows) to the partitions $\left(\lambda_{i}^{(w)}\right)^{*}$ and $\left(\lambda_{i}^{(w-1)}\right)^{*}$, which correspond to the $\beta$-sets $X_{\lambda_{i}^{(w)}}$ and $X_{\lambda_{i}^{(w-1)}}$. Indeed, these do have $p$-core $\gamma^{*}, p$-weight $w$ and $w-1$ respectively, and their hooks and hook-lengths correspond exactly to the unmixed bars and their lengths in $\lambda_{i}^{(w)}$ and $\lambda_{i}^{(w-1)}$. The fact that our definition of the $c_{j}$ 's differs from that of Malle and Navarro doesn't matter, since only the difference $c_{i}-c_{j}$ appears, and this doesn't depend on the definition used.

We now turn to the second part. The only mixed bars which are not common to $\lambda_{i}^{(w)}$ and $\lambda_{i}^{(w-1)}$ have lengths:

- in $\lambda_{i}^{(w)}:\left\{i+b_{i} p+p w+x_{k}^{(j)} \mid j \neq i, X_{j} \neq \emptyset, 0 \leq k \leq b_{j}\right\}$, where $x_{k}^{(j)}=j+k p$;

- in $\lambda_{i}^{(w-1)}:\left\{i+b_{i} p+p(w-1)+x_{k}^{(j)} \mid j \neq i, X_{j} \neq \emptyset, 0 \leq k \leq b_{j}\right\}$.

Since $i+b_{i} p+p w+x_{k}^{(j)}=i+b_{i} p+p(w-1)+x_{k+1}^{(j)}$, when we divide out, we're only left with $k=b_{j}$ in $\lambda_{i}^{(w)}$ and $k=0$ in $\lambda_{i}^{(w-1)}$. This yields

$$
\frac{\bar{H}_{m}\left(\lambda_{i}^{(w)}\right)}{\bar{H}_{m}\left(\lambda_{i}^{(w-1)}\right)}=\prod_{j \neq i, X_{j} \neq \emptyset} \frac{i+b_{i} p+p w+j+b_{j} p}{i+b_{i} p+p(w-1)+j}=\prod_{j \neq i, X_{j} \neq \emptyset} \frac{c_{i}+c_{j}+p w}{c_{i}+p(w-1)+j} .
$$


Corollary 3.2. If $X_{i} \neq \emptyset$, then

$$
\frac{\bar{H}\left(\lambda_{i}^{(w)}\right)}{\bar{H}\left(\lambda_{i}^{(w-1)}\right)}=p w \prod_{j \neq i, X_{j} \neq \emptyset}\left|p(w-1)+c_{i}-c_{j}\right|\left(p w+c_{i}+c_{j}\right) \prod_{X_{k}=X_{p-k}=\emptyset}\left|p w+c_{i}-k\right| .
$$

Proof. From Proposition 3.1, we easily obtain, if $X_{i} \neq \emptyset$,

$$
\frac{\bar{H}\left(\lambda_{i}^{(w)}\right)}{\bar{H}\left(\lambda_{i}^{(w-1)}\right)}=p w \prod_{0 \leq j \neq i \leq p-1}\left|p(w-1)+c_{i}-c_{j}\right| \prod_{j \neq i, X_{j} \neq \emptyset} \frac{c_{i}+c_{j}+p w}{c_{i}+p(w-1)+j}
$$

Separating the first product according to whether $X_{j}=\emptyset$ or not, we get

$$
p w \prod_{j \neq i, X_{j} \neq \emptyset}\left|p(w-1)+c_{i}-c_{j}\right|\left(p w+c_{i}+c_{j}\right) \prod_{k \neq i, X_{k}=\emptyset}\left|p(w-1)+c_{i}-c_{k}\right| \prod_{j \neq i, X_{j} \neq \emptyset} \frac{1}{p(w-1)+c_{i}+j} .
$$

Now, if $X_{j} \neq \emptyset$, then $X_{p-j}=\emptyset$ (since $\gamma$ is a $\bar{p}$-core), so that $p-j \neq i$ (since $\left.X_{i} \neq \emptyset\right)$, and $j=-[(p-j)-p]=-c_{p-j}$. We thus have

$$
\begin{aligned}
& \prod_{k \neq i, X_{k}=\emptyset}\left|p(w-1)+c_{i}-c_{k}\right| \prod_{j \neq i, X_{j} \neq \emptyset} \frac{1}{p(w-1)+c_{i}+j} \\
= & \prod_{k \neq i, X_{k}=\emptyset}\left|p(w-1)+c_{i}-c_{k}\right| \prod_{p-j \neq i, X_{j} \neq \emptyset, X_{p-j}=\emptyset} \frac{1}{p(w-1)+c_{i}-c_{p-j}} \\
= & \prod_{k \neq i, X_{k}=\emptyset, X_{p-k} \neq \emptyset} \frac{\left|p(w-1)+c_{i}-c_{k}\right|}{p(w-1)+c_{i}-c_{k}} \prod_{k \neq i, X_{k}=X_{p-k}=\emptyset}\left|p(w-1)+c_{i}-c_{k}\right| .
\end{aligned}
$$

If $X_{k}=\emptyset$, then $c_{k}=k-p<0$, so that $p(w-1)+c_{i}-c_{k}>0$ (since $c_{i} \geq 0$ ), whence the first product is 1 , and $p(w-1)+c_{i}-c_{k}=p w+c_{i}-k$. Finally, if $X_{k}=\emptyset$, then $k \neq i$. We therefore get, if $X_{i} \neq \emptyset$,

$$
\frac{\bar{H}\left(\lambda_{i}^{(w)}\right)}{\bar{H}\left(\lambda_{i}^{(w-1)}\right)}=p w \prod_{j \neq i, X_{j} \neq \emptyset}\left|p(w-1)+c_{i}-c_{j}\right|\left(p w+c_{i}+c_{j}\right) \prod_{X_{k}=X_{p-k}=\emptyset}\left|p w+c_{i}-k\right| .
$$

Remark: If $X_{i} \neq \emptyset$, then $c_{i} \geq i$, so that $c_{i}-k \geq i-k>-p$ and, whenever $w \geq 1$, we have $\left|p w+c_{i}-k\right|=p w+c_{i}-k$.

We now establish the analogous result for the bar-partitions $\lambda_{0}^{(w)}$ and $\lambda_{0}^{(w-1)}$ :

Proposition 3.3. With the above notation, we have 
- if $w>1$, then

$$
\frac{\bar{H}_{u}\left(\lambda_{0}^{(w)}\right)}{\bar{H}_{u}\left(\lambda_{0}^{(w-1)}\right)}=p w \prod_{0<j \leq p-1}\left|p(w-1)-c_{j}\right|
$$

and

$$
\frac{\bar{H}_{m}\left(\lambda_{0}^{(w)}\right)}{\bar{H}_{m}\left(\lambda_{0}^{(w-1)}\right)}=\prod_{j \neq 0, X_{j} \neq \emptyset} \frac{c_{j}+p w}{p(w-1)+j} ;
$$

- if $w=1$, then

$$
\frac{\bar{H}_{u}\left(\lambda_{0}^{(w)}\right)}{\bar{H}_{u}\left(\lambda_{0}^{(w-1)}\right)}=p \frac{\prod_{j \neq 0}\left|c_{j}\right|}{\prod_{1 \leq i \leq m} a_{i}}
$$

and

$$
\frac{\bar{H}_{m}\left(\lambda_{0}^{(w)}\right)}{\bar{H}_{m}\left(\lambda_{0}^{(w-1)}\right)}=\prod_{j \neq 0, X_{j} \neq \emptyset} \prod_{0 \leq k \leq b_{j}}(p+j+k p)=\prod_{1 \leq i \leq m}\left(a_{i}+p\right) .
$$

Proof. We start with the unmixed bars. All the unmixed bars $(x, y)$ in $\lambda_{0}^{(w)}$ such that $x \not \equiv 0(\bmod p)$ and $y \not \equiv 0(\bmod p)$ are also in $\lambda_{0}^{(w-1)}$, and conversely. Hence we just need to consider the unmixed bars $(x, y)$ with $x \equiv 0(\bmod p)$ or $y \equiv 0(\bmod p)$.

Those with $x \equiv y \equiv 0(\bmod p)$ contribute exactly $p w$ to $\frac{\bar{H}_{u}\left(\lambda_{0}^{(w)}\right)}{\overline{H_{u}\left(\lambda_{0}^{(w-1)}\right)}}$.

Next suppose $y \equiv 0(\bmod p)$, and $x \equiv j(\bmod p)$, with $0<j \leq p-1$. The bar-lengths to consider are thus:

- in $\lambda_{0}^{(w)}:\left\{p w-x_{k}^{(j)} \mid j \neq 0, b_{j} \leq w-2,1 \leq k \leq w-b_{j}-1\right\}$, where $x_{k}^{(j)}=j+b_{j} p+k p$;

- in $\lambda_{0}^{(w-1)}:\left\{p(w-1)-x_{k}^{(j)} \mid j \neq 0, b_{j} \leq w-3,1 \leq k \leq w-b_{j}-2\right\}$. These only appear if $w>1$; however, since, when $w=1,\left\{j \mid b_{j} \leq w-3\right\}=\emptyset$, the result applies in this case too.

Now, for any $j \neq 0$ such that $b_{j} \leq w-3$, we have $p w-x_{k}^{(j)}=p(w-1)-x_{k-1}^{(j)}$ for all $1 \leq k \leq w-b_{j}-1$, so that everything cancels out in $\frac{\bar{H}_{u}\left(\lambda_{0}^{(w)}\right)}{\bar{H}_{u}\left(\lambda_{0}^{(w-1)}\right)}$, except for $k=1$. We're thus left exactly with $p w-x_{1}^{(j)}=p(w-1)-c_{j}$. If, on the other hand, $b_{j}=w-2$, then the only bar that appears is given by $k=1$ in $\lambda_{0}^{(w)}$, and it contributes $p(w-1)-c_{j}$ to $\frac{\bar{H}_{u}\left(\lambda_{0}^{(w)}\right)}{\bar{H}_{u}\left(\lambda_{0}^{(w-1)}\right)}$.

Finally, suppose $x \equiv 0(\bmod p)$, and $y \equiv j(\bmod p)$, with $0<j \leq p-1$. Now, for $X \in\left\{X_{\lambda_{0}^{(w)}}, X_{\lambda_{0}^{(w-1)}}\right\}$, we must have $x=p r \notin X, y=j+k p \in X$, whence $k \leq b_{j}$, and $y>x$, so that $k \geq r$. Hence we just need to exclude $r=w$ in $\lambda_{0}^{(w)}$ (since $w p \in X_{\lambda_{0}^{(w)}}$ ), and $r=w-1$ in $\lambda_{0}^{(w-1)}$ (since $(w-1) p \in X_{\lambda_{0}^{(w-1)}}$ ), except if $w=1$ (in which case $\left.x=(w-1) p=0 \notin X_{\lambda_{0}^{(w-1)}}=X_{\gamma}\right)$. We thus get, for the product of these bar-lengths, 
- in $\lambda_{0}^{(w)}: \prod_{r \geq 0, r \neq w} \prod_{r \leq k \leq b_{j}\left(b_{j} \geq r\right)}(j+k p-p r)$;

- in $\lambda_{0}^{(w-1)}: \prod_{r \geq 0, r \neq w-1 \text { if } w>1} \prod_{r \leq k \leq b_{j}\left(b_{j} \geq r\right)}(j+k p-p r)$.

After cancellations (corresponding to fixed $r \notin\{w, w-1\}$ ), we're left with

- in $\lambda_{0}^{(w)}: \prod_{w-1 \leq k \leq b_{j}\left(b_{j} \geq w-1\right)}(j+k p-p(w-1))$;

- in $\lambda_{0}^{(w-1)}: \prod_{w \leq k \leq b_{j}\left(b_{j} \geq w\right)}(j+k p-p w)$,

and also, if $w=1, \prod_{w-1 \leq k \leq b_{j}\left(b_{j} \geq w-1\right)}(j+k p-p(w-1))$.

After further cancellations, we see that, whether $b_{j} \geq w$ or $b_{j}=w-1$, we're only left with $k=b_{j}$ in $\lambda_{0}^{(w)}$, which contributes $j+b_{j} p-p(w-1)=c_{j}-p(w-1)$ to $\frac{\bar{H}_{u}\left(\lambda_{0}^{(w)}\right)}{\bar{H}_{u}\left(\lambda_{0}^{(w-1)}\right)}$. In addition to this, and only in the case $w=1$, we have, left in $\lambda_{0}^{(w-1)}, \prod_{w-1 \leq k \leq b_{j}\left(b_{j} \geq w-1\right)}(j+$ $k p-p(w-1))=\prod_{0 \leq k \leq b_{j}\left(b_{j} \geq 0\right)}(j+k p)=\prod_{1 \leq i \leq m} a_{i}$.

Putting together the three cases for the values of $x$ and $y$, we obtain the announced expressions for $\frac{\bar{H}_{u}\left(\lambda_{0}^{(w)}\right)}{\bar{H}_{u}\left(\lambda_{0}^{(w-1)}\right)}$.

Turning now to mixed bars, we see that the only ones which are not common to $\lambda_{0}^{(w)}$ and $\lambda_{0}^{(w-1)}$ have lengths

- in $\lambda_{0}^{(w)}:\left\{p w+x_{k}^{(j)} \mid j \neq 0, X_{j} \neq \emptyset, 0 \leq k \leq b_{j}\right\}$, where $x_{k}^{(j)}=j+k p$;

- in $\lambda_{0}^{(w-1)}$, only if $w>1:\left\{p(w-1)+x_{k}^{(j)} \mid j \neq 0, X_{j} \neq \emptyset, 0 \leq k \leq b_{j}\right\}$.

If $w>1$, then $p w+x_{k}^{(j)}=p(w-1)+x_{k+1}^{(j)}$, so that we're only left with $k=b_{j}$ in $\lambda_{0}^{(w)}$ and $k=0$ in $\lambda_{0}^{(w-1)}$. This yields

$$
\frac{\bar{H}_{m}\left(\lambda_{0}^{(w)}\right)}{\bar{H}_{m}\left(\lambda_{0}^{(w-1)}\right)}=\prod_{j \neq 0, X_{j} \neq \emptyset} \frac{p w+j+b_{j} p}{p(w-1)+j} .
$$

If $w=1$, then we obtain

$$
\frac{\bar{H}_{m}\left(\lambda_{0}^{(w)}\right)}{\bar{H}_{m}\left(\lambda_{0}^{(w-1)}\right)}=\prod_{j \neq 0, X_{j} \neq \emptyset} \prod_{0 \leq k \leq b_{j}}(p+j+k p)=\prod_{1 \leq i \leq m}\left(a_{i}+p\right),
$$

as announced. 


\section{Corollary 3.4.}

$$
\frac{\bar{H}\left(\lambda_{0}^{(w)}\right)}{\bar{H}\left(\lambda_{0}^{(w-1)}\right)}= \begin{cases}p w \prod_{j \neq 0}\left|p(w-1)-c_{j}\right| \prod_{j \neq 0} \frac{p w+c_{j}}{p(w-1)+j} & \text { if } w>1 \\ p \prod_{j \neq 0}\left|c_{j}\right| \prod_{1 \leq i \leq m} \frac{a_{i}+p}{a_{i}} & \text { if } w=1\end{cases}
$$

We can now show that it's always possible to construct two bar-partitions with same bar-core $\gamma \neq \emptyset$ and weight $w \geq 2$ such that the corresponding products of bar-lengths are distinct.

Theorem 3.5. Suppose there exist $i_{1} \neq i_{2}$ such that $i_{1}, i_{2}>0, X_{i_{1}} \neq \emptyset$ and $X_{i_{2}} \neq \emptyset$, and suppose $c_{i_{1}}>c_{i_{2}}>c_{j}$ for all $j \notin\left\{i_{1}, i_{2}\right\}$. Then, whenever $w \geq 2$, we have $\bar{H}\left(\lambda_{i_{1}}^{(w)}\right)>$ $\bar{H}\left(\lambda_{i_{2}}^{(w)}\right)$. If, on the other hand, there exists a unique $i \neq 0$ such that $X_{i} \neq \emptyset$, then, whenever $w \geq 1$, we have $\bar{H}\left(\lambda_{i}^{(w)}\right)>\bar{H}\left(\lambda_{0}^{(w)}\right)$.

Proof. First suppose $i_{1}, i_{2}>0, X_{i_{1}} \neq \emptyset, X_{i_{2}} \neq \emptyset$, and $c_{i_{1}}>c_{i_{2}}>c_{j}$ for all $j \notin\left\{i_{1}, i_{2}\right\}$. We use Corollary 3.2.

Whenever $X_{k}=X_{p-k}=\emptyset$, we have $k \notin\left\{i_{1}, i_{2}\right\}$, and $p w+c_{i_{1}}-k>p w+c_{i_{2}}-k>0$ as soon as $w \geq 1$. Thus

$$
\prod_{X_{k}=X_{p-k}=\emptyset}\left(p w+c_{i_{1}}-k\right)>\prod_{X_{k}=X_{p-k}=\emptyset}\left(p w+c_{i_{2}}-k\right) .
$$

If, on the other hand, $j \notin\left\{i_{1}, i_{2}\right\}$ and $X_{j} \neq \emptyset$, then $0<c_{j}<c_{i_{1}}<c_{i_{2}}$. Hence $c_{i_{1}}+$ $c_{j}, c_{i_{2}}+c_{j}, c_{i_{1}}-c_{j}, c_{i_{2}}-c_{j}>0$. Thus $0<p w+c_{i_{2}}+c_{j}<p w+c_{i_{1}}+c_{j}$, and

$$
\left|p(w-1)+c_{i_{2}}-c_{j}\right|=p(w-1)+c_{i_{2}}-c_{j}<p(w-1)+c_{i_{1}}-c_{j}=\left|p(w-1)+c_{i_{1}}-c_{j}\right|,
$$

whence

$$
\left|p(w-1)+c_{i_{1}}-c_{j}\right|\left(p w+c_{i_{1}}+c_{j}\right)>\left|p(w-1)+c_{i_{2}}-c_{j}\right|\left(p w+c_{i_{2}}+c_{j}\right) .
$$

Finally, $c_{i_{1}}-c_{i_{2}}>0$, so that $\left|p(w-1)+c_{i_{1}}-c_{i_{2}}\right|=p(w-1)+c_{i_{1}}-c_{i_{2}}$ and

$$
\left|p(w-1)+c_{i_{1}}-c_{i_{2}}\right|\left(p w+c_{i_{1}}+c_{i_{2}}\right) \geq\left|p(w-1)+c_{i_{2}}-c_{i_{1}}\right|\left(p w+c_{i_{2}}+c_{i_{1}}\right),
$$

and this last inequality is in fact strict unless $w=1$, in which case the spin block we consider has abelian defect.

We thus obtain

$\prod_{j \neq i_{1}, X_{j} \neq \emptyset}\left|p(w-1)+c_{i_{1}}-c_{j}\right|\left(p w+c_{i_{1}}+c_{j}\right)>\prod_{j \neq i_{2}, X_{j} \neq \emptyset}\left|p(w-1)+c_{i_{2}}-c_{j}\right|\left(p w+c_{i_{2}}+c_{j}\right)$,

whence

$$
\frac{\bar{H}\left(\lambda_{i_{1}}^{(w)}\right)}{\bar{H}\left(\lambda_{i_{1}}^{(w-1)}\right)} \geq \frac{\bar{H}\left(\lambda_{i_{2}}^{(w)}\right)}{\bar{H}\left(\lambda_{i_{2}}^{(w-1)}\right)} \quad \text { whenever } w \geq 1
$$


and this inequality is strict whenever $w \geq 2$. Since $\lambda_{i_{1}}^{(0)}=\lambda_{i_{2}}^{(0)}=\gamma$, induction on $w$ gives that $\bar{H}\left(\lambda_{i_{1}}^{(w)}\right)>\bar{H}\left(\lambda_{i_{2}}^{(w)}\right)$ if $w \geq 2$.

Suppose now that there exists a unique $i \neq 0$ such that $X_{i} \neq \emptyset$. Then $X=X_{i}=$ $\left\{i+k p, 0 \leq k \leq b_{i}\right\}=\left\{a_{m}, \ldots, a_{1}\right\}=\left\{i, i+p, \ldots, c_{i}\right\}$. Also, $c_{i}=i+b_{i} p \geq i$, and $c_{j}=j-p$ whenever $j \neq i$. By Proposition 3.1, we have, whenever $w \geq 1$,

$$
\begin{aligned}
\frac{\bar{H}\left(\lambda_{i}^{(w)}\right)}{\bar{H}\left(\lambda_{i}^{(w-1)}\right)} & =p w \prod_{0 \leq j \neq i \leq p-1}\left|p(w-1)+c_{i}-(j-p)\right|(\text { the other product being } 1) \\
& =p w \prod_{0 \leq j \neq i \leq p-1}\left|p w+c_{i}-j\right|=p w \prod_{0 \leq j \neq i \leq p-1}\left(p w+c_{i}-j\right) .
\end{aligned}
$$

On the other hand, by Corollary 3.4, we have, if $w=1$,

$$
\begin{aligned}
\frac{\bar{H}\left(\lambda_{0}^{(w)}\right)}{\bar{H}\left(\lambda_{0}^{(w-1)}\right)} & =p \prod_{j \neq 0}\left|c_{j}\right| \prod_{1 \leq k \leq m} \frac{a_{k}+p}{a_{k}}=p\left(\prod_{j \neq 0}|j-p|\right) \frac{c_{i}+p}{i} \\
& =p\left(\prod_{1 \leq j \leq p-1}(p-j)\right) \frac{c_{i}+p}{i}=p(p-1) ! \frac{c_{i}+p}{i}=p ! \frac{c_{i}+p}{i}
\end{aligned}
$$

while, if $w>1$, then we have

$$
\begin{aligned}
\frac{\bar{H}\left(\lambda_{0}^{(w)}\right)}{\bar{H}\left(\lambda_{0}^{(w-1)}\right)} & =p w \prod_{j \neq 0}\left|p(w-1)-c_{j}\right| \text { (the other product being 1) } \\
& =p w\left|p(w-1)-c_{i}\right| \prod_{1 \leq j \neq i \leq p-1}|p(w-1)-(j-p)| \\
& =p w\left|p(w-1)-c_{i}\right| \prod_{1 \leq j \neq i \leq p-1}(p w-j)
\end{aligned}
$$

If $w>1$, then, whenever $1 \leq j \neq i \leq p-1$, we have $p w-j<p w+c_{i}-j$, and (for $j=0$ ) $\left|p(w-1)-c_{i}\right|<p(w-1)+c_{i}<p w+c_{i}-0$. Hence, in this case,

$$
\frac{\bar{H}\left(\lambda_{i}^{(w)}\right)}{\bar{H}\left(\lambda_{i}^{(w-1)}\right)}>\frac{\bar{H}\left(\lambda_{0}^{(w)}\right)}{\bar{H}\left(\lambda_{0}^{(w-1)}\right)}
$$

If $w=1$, then

$$
\frac{\bar{H}\left(\lambda_{i}^{(1)}\right)}{\bar{H}\left(\lambda_{i}^{(0)}\right)}=p \prod_{0 \leq j \neq i \leq p-1}\left(p+c_{i}-j\right)=p\left(p+c_{i}\right) \prod_{1 \leq j \neq i \leq p-1}\left(p+c_{i}-j\right) .
$$

Now $\prod_{1 \leq j \neq i \leq p-1}\left(p+c_{i}-j\right) \geq \prod_{1 \leq j \neq i \leq p-1}(p+i-j)$ (since $\left.c_{i} \geq i>0\right)$. We rewrite $\prod_{1 \leq j \neq i \leq p-1}(p+i-j)$ as $\prod_{1 \leq j \leq i-1}(p+i-j) \prod_{i+1 \leq j \leq p-1}(p+i-j)$. Then $\prod_{1 \leq j \leq i-1}(p+i-j)=$ $(p+1)(p+2) \cdots(p+i-1)>1.2 \cdots(i-1) \stackrel{=}{=}(i-1)$ !, and $\prod_{i+1 \leq j \leq p-1}(p+i-j)=$ 
$(p-1)(p-2) \cdots(p+i-(p-1))=\frac{(p-1) !}{i !}$. Hence $\prod_{1 \leq j \neq i \leq p-1}\left(p+c_{i}-j\right)>\frac{(i-1) !(p-1) !}{i !}=\frac{(p-1) !}{i}$ and

$$
\frac{\bar{H}\left(\lambda_{i}^{(1)}\right)}{\bar{H}\left(\lambda_{i}^{(0)}\right)}>p\left(p+c_{i}\right) \frac{(p-1) !}{i}=p ! \frac{p+c_{i}}{i}=\frac{\bar{H}\left(\lambda_{0}^{(1)}\right)}{\bar{H}\left(\lambda_{0}^{(0)}\right)} .
$$

Since $\lambda_{i}^{(0)}=\lambda_{0}^{(0)}=\gamma$, induction on $w$ yields that $\bar{H}\left(\lambda_{i}^{(w)}\right)>\bar{H}\left(\lambda_{0}^{(w)}\right)$ whenever $w \geq 1$.

Finally, we deal with the case of the principal spin-blocks, that is the spin-blocks of 2. $\mathfrak{S}_{n}$ and $2 . \mathfrak{A}_{n}$ labelled by the empty bar-core.

Proposition 3.6. For any $w \geq 2$ and odd prime $p$, the bar-partitions $\mu_{0}^{(w)}=($ pw) and $\mu_{1}^{(w)}=(p w-1,1)$ of pw satisfy $\bar{H}\left(\mu_{0}^{(w)}\right)>2 \bar{H}\left(\mu_{1}^{(w)}\right)$.

Proof. This is obvious, since the bar-lengths in $\mu_{0}^{(w)}$ are $\{1,2, \ldots, p w-1, p w\}$, while those in $\mu_{1}^{(w)}$ are $\{1,2, \ldots, p w-3, p w-1, p w, 1\}$, and, since $w \geq 2$ and $p \geq 3$, we have $p w-2>2$.

\section{Height zero spin-characters of $2 . \mathfrak{S}_{n}$ and $2 . \mathfrak{A}_{n}$}

We can now prove our main result

Theorem 4.1. Let $n \geq 4$ be any integer and $p$ be an odd prime. If $B$ is a spin p-block of $2 . \mathfrak{A}_{n}$ with non-abelian defect groups, then $B$ contains two height 0 characters which have distinct degrees.

Proof. Let $\gamma$ be the $\bar{p}$-core labelling $B$ and the corresponding spin $p$-block $B^{*}$ of $2 . \mathfrak{S}_{n}$. Let $w$ be the $\bar{p}$-weight of $B$ and $B^{*}$. Since $B$ has non-abelian defect, we have $w \geq p$. We use the notation of Section 3.

First suppose that $\gamma=\emptyset$, so that $n=p w$. Take any two spin-characters $\chi_{0}$ and $\chi_{1}$ of $2 . \mathfrak{S}_{n}$ labelled respectively by the bar-partitions $\mu_{0}^{(w)}=(p w)$ and $\mu_{1}^{(w)}=(p w-1,1)$, and take spin-characters $\psi_{0}$ and $\psi_{1}$ appearing in the restrictions to $2 . \mathfrak{A}_{n}$ of $\chi_{0}$ and $\chi_{1}$ respectively. In particular, we have $\psi_{0}, \psi_{1} \in \operatorname{Irr}_{0}(B)$. We have

$$
\chi_{0}(1)=2^{\lfloor(n-1) / 2)\rfloor} \frac{n !}{\bar{H}\left(\mu_{0}^{(w)}\right)} \text { and } \chi_{1}(1)=2^{\lfloor(n-2) / 2)\rfloor} \frac{n !}{\bar{H}\left(\mu_{1}^{(w)}\right)} .
$$

First suppose that $n=p w$ is odd. Then $\sigma\left(\mu_{0}^{(w)}\right)=1$ and $\sigma\left(\mu_{1}^{(w)}\right)=-1$, so that, when restricted to $2 . \mathfrak{A}_{n}, \chi_{0}$ splits while $\chi_{1}$ doesn't. We thus have $\psi_{0}(1)=\frac{1}{2} \chi_{0}(1)$ and $\psi_{1}(1)=\chi_{1}(1)$. Since, in this case, $\left.\left.\lfloor(n-2) / 2)\right\rfloor=\lfloor(n-1) / 2)\right\rfloor-1$, we have, by Proposition 3.6

$$
\frac{1}{2} \chi_{0}(1)=\frac{2^{\lfloor(n-1) / 2)\rfloor-1} n !}{\bar{H}\left(\mu_{0}^{(w)}\right)}=\frac{2^{\lfloor(n-2) / 2)\rfloor} n !}{\bar{H}\left(\mu_{0}^{(w)}\right)}<\frac{2^{\lfloor(n-2) / 2)\rfloor} n !}{\bar{H}\left(\mu_{1}^{(w)}\right)}=\chi_{1}(1),
$$

so that $\psi_{0}, \psi_{1} \in \operatorname{Irr}_{0}(B)$ satisfy $\psi_{0}(1)<\psi_{1}(1)$. 
Suppose now that $n$ is even. In this case, when restricted to $2 . \mathfrak{A}_{n}, \chi_{1}$ splits while $\chi_{0}$ doesn't, and $\lfloor(n-2) / 2)\rfloor=\lfloor(n-1) / 2)\rfloor$. By Proposition 3.6, we get

$$
\psi_{0}(1)=\chi_{0}(1)=\frac{2^{\lfloor(n-1) / 2)\rfloor} n !}{\bar{H}\left(\mu_{0}^{(w)}\right)}<\frac{2^{\lfloor(n-2) / 2)\rfloor} n !}{2 \bar{H}\left(\mu_{1}^{(w)}\right)}=\frac{1}{2} \chi_{1}(1)=\psi_{1}(1),
$$

so that $\psi_{0}, \psi_{1} \in \operatorname{Irr}_{0}(B)$ satisfy $\psi_{0}(1)<\psi_{1}(1)$.

This proves that, whenever the principal spin $p$-block $B$ of $2 . \mathfrak{A}_{n}$ has weight $w \geq 2$ (in particular, when $B$ has non-abelian defect group), $B$ contains two height 0 characters with distinct degrees.

From now on, we therefore suppose that $\gamma \neq \emptyset$. If there exist $i \neq j$ such that $X_{i} \neq \emptyset$ and $X_{j} \neq \emptyset$, then we can suppose $c_{i}>c_{j}>c_{k}$ for all $k \notin\{i, j\}$, and consider the barpartitions $\lambda_{i}^{(w)}$ and $\lambda_{j}^{(w)}$ of $n$ constructed in Section 3. They have the same number of parts $m\left(\lambda_{i}^{(w)}\right)=m\left(\lambda_{j}^{(w)}\right)=m(\gamma)=m$. If $\chi_{i}$ and $\chi_{j}$ are spin-characters of $2 . \mathfrak{S}_{n}$ labelled by $\lambda_{i}^{(w)}$ and $\lambda_{j}^{(w)}$ respectively, then, by construction, $\chi_{i}$ and $\chi_{j}$ have $p$-height 0 , and, by Theorem 3.5

$$
\chi_{i}(1)=2^{\lfloor(n-m) / 2)\rfloor} \frac{n !}{\bar{H}\left(\lambda_{i}^{(w)}\right)}<2^{\lfloor(n-m) / 2)\rfloor} \frac{n !}{\bar{H}\left(\lambda_{j}^{(w)}\right)}=\chi_{j}(1) .
$$

Also, $\sigma\left(\lambda_{i}^{(w)}\right)=\sigma\left(\lambda_{j}^{(w)}\right)$, so that both $\chi_{i}$ and $\chi_{j}$ split when restricted to $2 . \mathfrak{A}_{n}$, or none of them does. In both cases, we thus obtain $\psi_{i}, \psi_{j} \in \operatorname{Irr}_{0}(B)$ which satisfy $\psi_{i}(1)<\psi_{j}(1)$.

Now suppose there exists a unique $i$ such that $X_{i} \neq \emptyset$, and consider the bar-partitions $\lambda_{i}^{(w)}$ and $\lambda_{0}^{(w)}$ of $n$. Then $m\left(\lambda_{i}^{(w)}\right)=m(\gamma)=m$ and $m\left(\lambda_{0}^{(w)}\right)=m+1$. If $\chi_{i}$ and $\chi_{0}$ are spin-characters of $2 . \mathfrak{S}_{n}$ labelled by $\lambda_{i}^{(w)}$ and $\lambda_{0}^{(w)}$ respectively, then, by construction, $\chi_{i}$ and $\chi_{0}$ have $p$-height 0 , and we have

$$
\chi_{i}(1)=2^{\lfloor(n-m) / 2)\rfloor} \frac{n !}{\bar{H}\left(\lambda_{i}^{(w)}\right)} \text { and } \chi_{0}(1)=2^{\lfloor(n-m-1) / 2)\rfloor} \frac{n !}{\bar{H}\left(\lambda_{0}^{(w)}\right)} .
$$

First suppose that $n-m$ is even, so that $\sigma\left(\lambda_{i}^{(w)}\right)=1$ and $\sigma\left(\lambda_{0}^{(w)}\right)=-1$. When restricted to $2 . \mathfrak{A}_{n}, \chi_{i}$ thus splits, while $\chi_{0}$ doesn't. Taking characters $\psi_{i}$ and $\psi_{0}$ in these restrictions, we obtain, by Theorem 3.5, and since in this case $\lfloor(n-m-1) / 2)\rfloor=$ $\lfloor(n-m) / 2)\rfloor-1$,

$$
\frac{1}{2} \chi_{i}(1)=\frac{2^{\lfloor(n-m) / 2)\rfloor-1} n !}{\bar{H}\left(\lambda_{i}^{(w)}\right)}=\frac{2^{\lfloor(n-m-1) / 2)\rfloor} n !}{\bar{H}\left(\lambda_{i}^{(w)}\right)}<\frac{2^{\lfloor(n-m-1) / 2)\rfloor} n !}{\bar{H}\left(\lambda_{0}^{(w)}\right)}=\chi_{0}(1),
$$

so that $\psi_{i}, \psi_{0} \in \operatorname{Irr}_{0}(B)$ satisfy $\psi_{i}(1)<\psi_{0}(1)$.

Suppose now that $n-m$ is odd, so that $\sigma\left(\lambda_{i}^{(w)}\right)=-1$ and $\sigma\left(\lambda_{0}^{(w)}\right)=1$. When restricted to $2 . \mathfrak{A}_{n}, \chi_{0}$ thus splits, while $\chi_{i}$ doesn't. Taking characters $\psi_{0}$ and $\psi_{i}$ in these restrictions, we have

$$
\psi_{i}(1)=\chi_{i}(1)=2^{\lfloor(n-m) / 2)\rfloor} \frac{n !}{\bar{H}\left(\lambda_{i}^{(w)}\right)},
$$


and, since in this case $\lfloor(n-m-1) / 2)\rfloor=\lfloor(n-m) / 2)\rfloor$,

$$
\psi_{0}(1)=\frac{1}{2} \chi_{0}(1)=\frac{2^{\lfloor(n-m-1) / 2)\rfloor} n !}{2 \bar{H}\left(\lambda_{0}^{(w)}\right)}=\frac{2^{\lfloor(n-m) / 2)\rfloor} n !}{2 \bar{H}\left(\lambda_{0}^{(w)}\right)} .
$$

We therefore obtain $\psi_{0}(1) \neq \psi_{i}(1)$, unless $\bar{H}\left(\lambda_{i}^{(w)}\right)=2 \bar{H}\left(\lambda_{0}^{(w)}\right)$.

To exclude this last possibility, write $\bar{H}\left(\lambda_{i}^{(w)}\right)=\bar{H}_{p}\left(\lambda_{i}^{(w)}\right) \bar{H}_{p^{\prime}}\left(\lambda_{i}^{(w)}\right)$ and $\bar{H}\left(\lambda_{0}^{(w)}\right)=$ $\bar{H}_{p}\left(\lambda_{0}^{(w)}\right) \bar{H}_{p^{\prime}}\left(\lambda_{0}^{(w)}\right)$. Since $\chi_{i}$ and $\chi_{0}$ both have $p$-height 0 , we have $\bar{H}_{p}\left(\lambda_{i}^{(w)}\right)=\bar{H}_{p}\left(\lambda_{0}^{(w)}\right)$. Also, since all the $(p)$-bars in $\lambda_{i}^{(w)}$ and $\lambda_{0}^{(w)}$ are of type 1 or of type 2, Proposition 2.5 in [2] gives $\bar{H}_{p^{\prime}}\left(\lambda_{i}^{(w)}\right) \equiv \pm \bar{H}(\gamma)(\bmod p)$ and $\bar{H}_{p^{\prime}}\left(\lambda_{0}^{(w)}\right) \equiv \pm \bar{H}(\gamma)(\bmod p)$. If $\bar{H}\left(\lambda_{i}^{(w)}\right)=$ $2 \bar{H}\left(\lambda_{0}^{(w)}\right)$, and since $\bar{H}(\gamma)$ is invertible $(\bmod p)$, this implies $1 \equiv \pm 2(\bmod p)$. This can only happen if $p=3$, in which case $1 \equiv-2(\bmod p)$.

We therefore suppose that $p=3$, and we first suppose that $c_{i} \geq i+p$. Looking at the case $w=1$ in the proof of Theorem 3.5, we see that, writing $\{1,2\} \backslash\{i\}=\{j\}$, we have

$$
\frac{\bar{H}\left(\lambda_{i}^{(1)}\right)}{\bar{H}(\gamma)}=3\left(3+c_{i}\right)\left(3+c_{i}-j\right) \text { and } \frac{\bar{H}\left(\lambda_{0}^{(1)}\right)}{\bar{H}(\gamma)}=3\left(3+c_{i}\right) \frac{2}{i}
$$

But since $c_{i} \geq i+p$, we have $3+c_{i}-j \geq 6+i-j \in\{5,7\}$, so that $3+c_{i}-j>\frac{2}{i}$ and $\bar{H}\left(\lambda_{i}^{(1)}\right)>2 \bar{H}\left(\lambda_{0}^{(1)}\right)$. Since, for each $2 \leq k \leq w$, we have $\frac{\bar{H}\left(\lambda_{i}^{(k)}\right)}{\bar{H}\left(\lambda_{i}^{(k-1)}\right)}>\frac{\bar{H}\left(\lambda_{0}^{(k)}\right)}{\bar{H}\left(\lambda_{0}^{(k-1)}\right)}$, we finally obtain that, if $p=3$ and $c_{i} \geq i+p$, then $\bar{H}\left(\lambda_{i}^{(w)}\right)>2 \bar{H}\left(\lambda_{0}^{(w)}\right)$. Thus, in this case, we get, as above, $\psi_{i}, \psi_{0} \in \operatorname{Irr}_{0}(B)$ such that $\psi_{0}(1) \neq \psi_{i}(1)$.

The last case to study is thus when $p=3$ and $c_{i}=i$. In this case, we have $\gamma=(i)$, $\lambda_{i}^{(w)}=(p w+i)$ and $\lambda_{0}^{(w)}=(p w, i)$. The bar lengths in $\lambda_{i}^{(w)}$ are $\{1,2, \ldots, p w+i\}$, while those in $\lambda_{0}^{(w)}$ are $\{1, \ldots, p w-(i+1), p w-(i-1), \ldots, p w, p w+i, 1, \ldots, i\}$. We thus have

$$
\frac{\bar{H}\left(\lambda_{i}^{(w)}\right)}{\bar{H}\left(\lambda_{0}^{(w)}\right)}=\left\{\begin{array}{ll}
p w-1 & \text { if } i=1 \\
\frac{(p w+1)(p w-2)}{2} & \text { if } i=2
\end{array} .\right.
$$

Since $p=3$ and $w \geq 2$, we obtain in both cases that $\bar{H}\left(\lambda_{i}^{(w)}\right)>2 \bar{H}\left(\lambda_{0}^{(w)}\right)$. Thus, in this case also, we get $\psi_{i}, \psi_{0} \in \operatorname{Irr}_{0}(B)$ such that $\psi_{0}(1) \neq \psi_{i}(1)$.

Finally, we have shown that, if there exists a unique $i$ such that $X_{i} \neq \emptyset$, then we can find $\psi_{i}, \psi_{0} \in \operatorname{Irr}_{0}(B)$ such that $\psi_{0}(1) \neq \psi_{i}(1)$. This ends the proof.

Corollary 4.2. Let $n \geq 4$ be any integer and $p$ be a prime. If $B$ is a p-block of $2 . \mathfrak{A}_{n}$ all of whose height zero characters have the same degree, then $B$ is nilpotent.

Proof. As we mentionned in Section 2, if $p=2$ or if $p$ is odd and $B$ is a non-faithful block, then $B$ must have cyclic defect groups. Since Brauer's Height Zero Conjecture is obvious in this case (as all the characters are linear), [3, Theorem 4.1] implies that $B$ is nilpotent.

If $p$ is odd and $B$ is a spin-block, then, by Theorem $4.1, B$ must have abelian defect groups. Since Brauer's Height Zero Conjecture holds for $2 . \mathfrak{A}_{n}$ for $p$ odd (see [4]), we deduce from [3, Theorem 4.1] that $B$ is nilpotent. 


\section{References}

[1] M. Broué and L. Puig. A Frobenius theorem for blocks. Invent. Math., 56:117-128, 1980.

[2] J.-B. Gramain. The Isaacs-Navarro Conjecture for covering groups of the symmetric and alternating groups in odd characteristic. J. Algebraic Combinatorics, 34(3):401426, 2011

[3] G. Malle and G Navarro. Blocks with equal height zero degrees. Trans. Amer. Math. Soc., 363:6647-6669, 2011.

[4] J. B. Olsson. On the $p$-blocks of symmetric and alternating groups and their covering groups. J. Algebra, 128:188-213, 1990.

[5] J. B. Olsson. Combinatorics and representations of finite groups. Vorlesungen aus dem Fachbereich Mathematik der Universität GH Essen, Heft 20, 1993.

[6] I. Schur. Über die Darstellung der symmetrischen und der alternierenden Gruppen durch gebrochente lineare Substitutionen. J. reine angew. Math., 139:155-250, 1911. 